\title{
Triboluminescence Flashes from High-Speed Ruptures in Carbon Nanotube Macro-Yarns
}

Thurid S. Gspann ${ }^{\mathrm{a}}$, Nigel H.H. Ngern ${ }^{\mathrm{b}}$, Andrew Fowler ${ }^{\mathrm{a}}$, Alan H. Windle ${ }^{\mathrm{a}}$, Vincent B.C. Tan ${ }^{\mathrm{b}}$, James A. Elliott ${ }^{\mathrm{a}}$

${ }^{a}$ Department of Materials Science and Metallurgy, University of Cambridge, 27 Charles Babbage Road, CB3 OFS Cambridge, UK

${ }^{b}$ Department of Mechanical Engineering, National University of Singapore, 9 Engineering Drive 1, Singapore 117576

During tensile tests of carbon nanotube (CNT) macrostructures (ribbons, ropes and tows) under dynamic strain rates $\left(1000 \mathrm{sec}^{-1}\right)$, we recorded temporally sporadic, spatially localised visible light emissions ("flashes") of less than $1.5 \mu$ s duration. The flashes occurred at the fracture sites and were, depending on the sample morphology, either distributed randomly over time (for tows) or occurred all at once over larger areas of several pixels (for ribbons). In situ thermal camera measurements, as well as ex situ analysis by electron microscopy reveal a hierarchical mechanism of overall heating over the whole sample length during straining, and localized heating around the fracture surfaces. Temperatures around the fracture tip were calculated to be of $1,800 \mathrm{~K}$ in average. We propose that the flashes are caused by charge separation due to CNT bond fracture and gas discharge of the surrounding gases. Triboluminescence, known for larger sugar crystals, has not been observed for carbon nanotubes previously. It results from the yarn-like morphology, the ultra-high strength and thermal conductivity of our CNT fibers, which at high strain rates concentrate the strain at CNT level and lead to CNT fracture, rather than bundle sliding.

Keywords. Carbon Nanotube, Failure Mode, Yarn Structure, Strain Rate Effect, Triboluminescence

Various macrostructures made from carbon nanotubes synthesised by direct spinning using a chemical vapour deposition (CVD) process [1] [2] were subjected to tensile tests with strain rates up to $1000 \mathrm{sec}^{-1}$. We observed bright bluish-white flashes being emitted at the position and time of fracture, and videos of the flashes can be found in Supporting Online Material. The samples investigated were (1) CNT ribbons laser-cut out of a film parallel to CNT bundle alignment, (2) films rolled into CNT ropes, and (3) CNT tows comprising several hundreds of individually-condensed microfibers (Figure S1). All samples have a yarn-like microstructure comprised of entangled CNT bundles. Further details of the macro-sample preparation and morphology can be

* Corresponding Author: Thurid Gspann, tsg28@cam.ac.uk 
found in [2] [3]. For the reference samples of the high strain rate tests, dry carbon fiber and Dyneema tows, no such emission was recorded and they will not be further discussed here in any more detail.

Having first observed the flashes with a high-speed camera of maximum 54,000 frames per second (fps) and then upgrading to $100,000 \mathrm{fps}$ (Photron Fastcam SA-Z), it became obvious that the effect was highly reproducible and that the majority of events had initially been simply missed at lower frame rate. The flashes last no longer than one frame recorded over $8 \cdot 10^{-5} \mathrm{sec}$, limiting their duration to less than $1.25 \mu \mathrm{s}$. We chose a slower strain-rate of $100 \mathrm{sec}^{-1}$ to increase the number of recorded frames during the entire fracture to increase the probability of capturing the emitted flash. We conducted our experiments with as little background illumination as possible to reduce reflection. The flashes occurred arbitrarily over the entire sample length on both the illuminated and shaded sides of the samples independent from ambient lighting.

Within the three sample types, CNT ropes have the largest bulk cross-section and most intense flashes recorded (Video S-V1). Measuring the flash was however hindered by the typical sheath-and-core fracture mechanism, with the sheath obscuring the flash within.
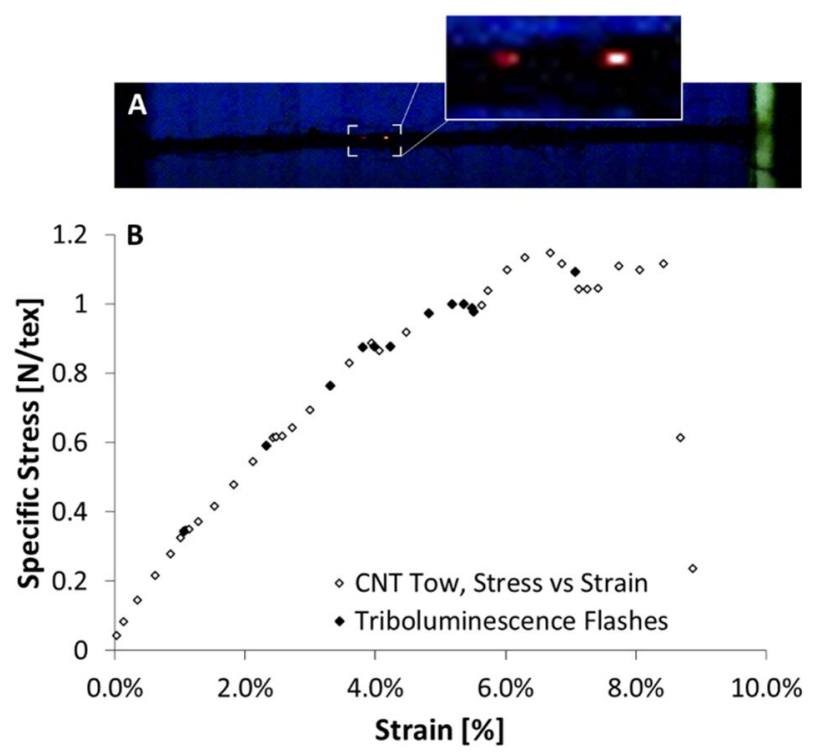

Figure 1: During high strain rate tensile tests, fracturing filaments in CNT tows emit bursts of luminescence flashes. (A) shows a high-speed camera frame capturing two flashes, further magnified in the inset. (B) The correlation of high speed camera, stress over time and strain over time recordings show that the majority of the flashes (black diamond symbol) occur at the beginning of plastic deformation. 
CNT tows offered the highest probability of capturing flashes as the elements break in succession, each break being likely to produce a flash (Video S-V2, and 3) of one pixel in size, $27 \times 27 \mu \mathrm{m}^{2}$. The majority of the events concentrate at the beginning of plastic deformation, with only few filaments breaking in the elastic zone prestretched and strained first because of unequal alignment by the manual sample preparation.

Ribbons, $3 \mathrm{~mm}$ wide, presented the largest area for observation and when mounted at 45 degrees allowed correlating measurements from two cameras. Fracture here occurs by a crack progressing by rupture through the film, and the recorded flashes here could span over several pixels. The ribbon in Figure 2 (and Video S-V4) shows a multitude of simultaneous flashes at the fracture line, with visible colours ranging from orange-yellow to bluish-white. The temperature of the flashes could not be directly determined from the image's RGB values as there is a many-to-one mapping between a known electromagnetic frequency spectrum and a particular RGB value via black body radiation, but not vice versa.

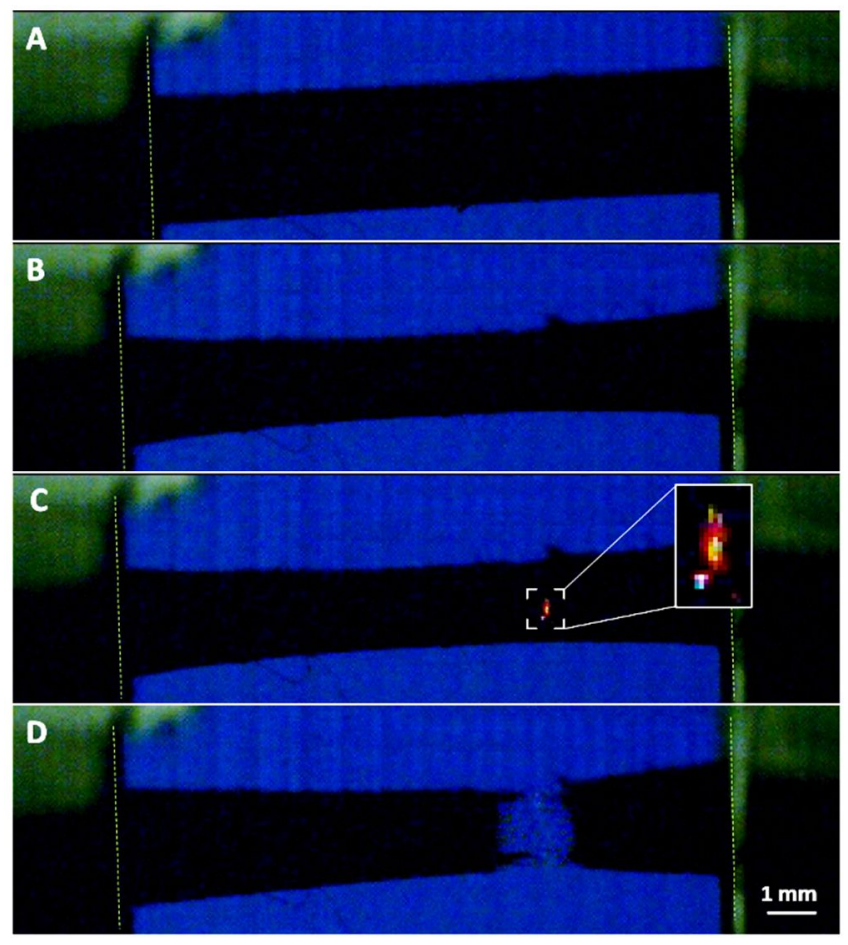

Figure 2: High-speed camera images of a CNT ribbon (A) before the dynamic tensile test, (B) one frame before the fracture, (C) the bright light emission at point of fracture, and (D) one frame after the flash showing the fractured halves pulling apart. The images were recorded at a frame rate of $100,000 \mathrm{fps}$. For simultaneous recording of optical and thermal image, the ribbons were mounted at $45^{\circ}$ which accounts for the upper edge of the ribbon not being in focus here. 
We used an IR camera (FLIR SC5500) to investigate sample heating during tensioning and fracture (Figure 3 and Video S-V5). Although the much lower frame rate of the thermal camera made a direct comparison impossible, we observed an overall temperature increase during tensioning and a localised increase up to $630 \mathrm{~K}$ at fracture. With a frame rate of $3.6 \mathrm{kHz}$, a $10 \mu$ s integration time and $180 \mu \mathrm{m}$ pixel resolution, we can assume that the actual temperature of the flashes, lasting much shorter and over a much smaller area, is significantly higher.

With the specific conductivity as measured for CNT microfibers [2], and a thermal diffusivity as by [4] [5], the decay time for a temperature of $1000 \mathrm{~K}$ would match the duration of one frame (Detailed calculation in SOM).

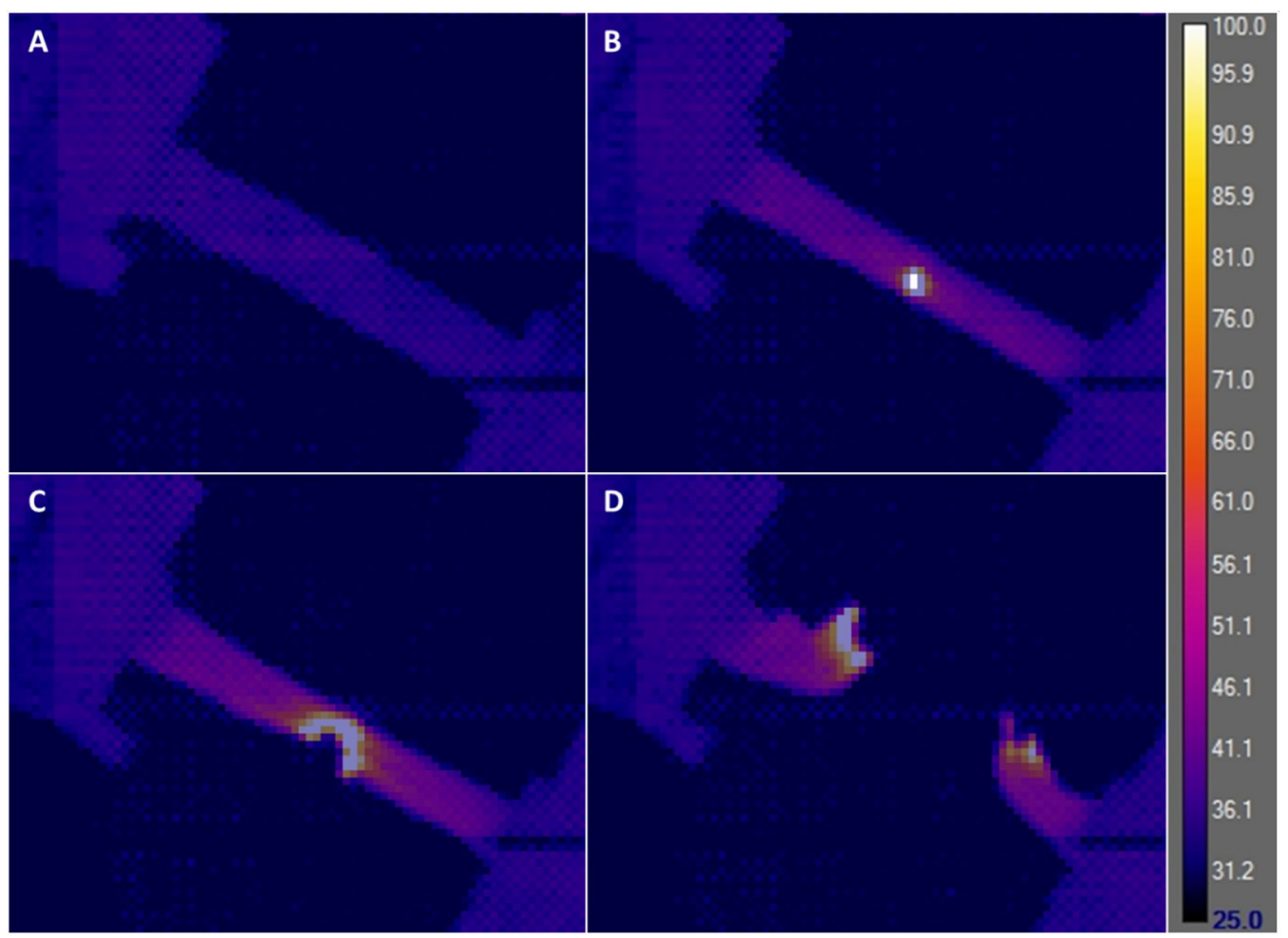

Figure 3: IR camera images (A) before the dynamic tensile test, (B) one frame before the fracture showing a localized heat increase, (C) during fracture, and (D) one frame after the fracture. For means of presentation, the colour bar is here limited to $25-100^{\circ} \mathrm{C}$, the maximum temperature measured in this sample was $226^{\circ} \mathrm{C}$.

Next, we assume that one flash event coincides with the IR camera pixel showing the highest temperature. By integrating the total emitted photon energy of a black body decaying linearly in temperature with time, weighted by the ratio of high-speed camera to IR camera pixel areas, we calculated initial temperatures of around $1800 \mathrm{~K}$ with maximum values of up to $4600 \mathrm{~K}$. The high deviation originates from the thermal camera's limited frame rate and large pixel size. 
Fracture surfaces of the high-strain rate samples display extraordinarily sharp edges (Figure 4), very different to the fibrous pull-out known from static tests (Figure S2), and we find evidence of a molten substance covering nanotube bundles and bridging the layers of a rope cross-section, possibly residue of the carbonaceous coating around the CNT bundles. Other samples (Figure S3) with less abrupt fracture-ends show a scorched region, coated in molten or burned material.

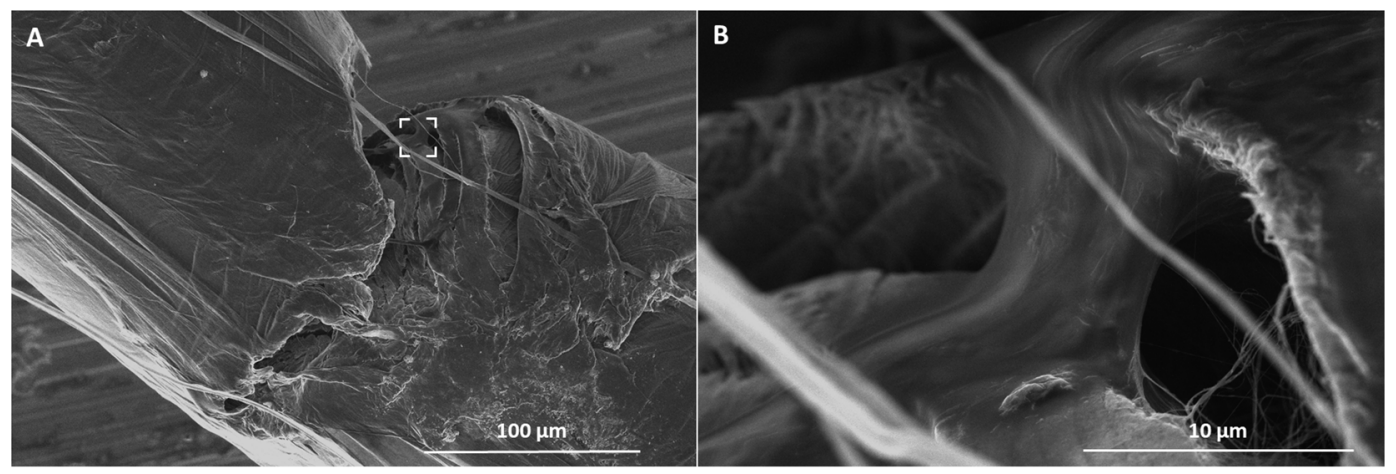

Figure 4: (A) An almost complete fracture of a rolled CNT rope showing (B) molten material covering CNT bundles and bridging the circular layers. Most significant difference to standard fracture of CNT structures is the absence of a pull-out zone. The fractured ends were remarkably sharp compared to the common fibrous pull-out.

Both cases unmistakably depict the consequence of strong localized heating around the crack area which concurs with the calculated temperatures of 1,000 to $2,000 \mathrm{~K}$. This temperature, although extensively high for being produced by friction, and certainly high enough for CNT combustion in air, is nowhere near the several thousand degrees required in solids to produce a bluish-white flash by black body radiation.

We therefore conclude that the observed flashes are caused by triboluminescence, more explicitly by charge separation caused by bond fracture, followed by ionization and recombination of the surrounding gas. Triboluminescence is defined as luminescence caused by mechanical deformation and is fundamentally different from black body radiation or combustion in that it does not require or produce heat. However, the effect often occurs simultaneously with heating close to the created crack area.

The effect has been shown for several crystals including sugar, aromatic acids and polymers [6] [7]. It has been shown that triboluminescence is caused by asymmetry, either by a non-centrosymmetric crystal structure, or impurities and defects in a centrosymmetric structure [7] [8]. Given their chirality, nanotubes fulfil the noncentrosymmetric criteria, and the samples contain a large amount of non-tubular, aromatic polymeric carbon adsorbed to the CNT bundles [2]. For a given triboluminescent material, the triboluminescence spectrum 
either resembles their solid-state photoluminescence, or resembles the discharge spectrum of the gases surrounding the sample, or a combination of both [8]. For carbon nanotubes, photoluminescence occurs in the NIR to MIR range, outside the visible spectrum [9]. On the other hand, the spectral gas discharge lines [10] of surrounding air, mainly nitrogen (78\%), oxygen $(21 \%)$ and small amounts of other gases $(<1 \%)$, agrees well with the observation of visible white light.

As known from low strain rate tensile tests, the intrinsic strength of carbon nanotubes is far higher than the friction between CNTs, and macro-sample fracture typically occurs by bundle pull-out. However, by means of the bundle entanglement and the co-synthesised carbonaceous bundle coating, an applied high strain rate at fiber level can be magnified manifold at bundle or CNT level by the network structure, as entanglement points lock up with not enough time to relax. The action of a small frictional force over large tangential lengths could well exceed the force required to cause bond breakage, so that macro-structure failure is caused by a combination of CNT fracture and sliding, rather than sliding alone.

Another sub-category of triboluminescence is the so-called tribo-induced thermoluminescence [11], where grinding (or radiation) of crystals would excite electrons which get trapped and are subsequently released by recombination with phonons when the crystals are heated. Thermoluminescence has been shown for carbon nanotubes although for comparatively low temperatures, $300-600 \mathrm{~K}$ [12], and could occur under the assumption that the friction between CNT bundles provides both the 'grinding' as well as the local heating needed. Here, we will use the term triboluminescent to refer to luminescence by mechanical deformation, conveniently including the term 'tribo-' (Greek 'to rub'), without further specifying whether the luminescence is caused by gas discharge or thermoluminescence.

As vacuum or inert gas enclosure of the whole test setup was not feasible, we coated the samples in a lowviscosity compound to inhibit the effects of direct contact with air: The samples were either soaked in acetone or coated in a UV-curable mixture of acrylates (" 5 Second Fix", Ontel Products Corp.) without curing the polymer. Both acetone and the uncured polymer remain liquid and coat the entire sample's surface during the test, the latter for significantly longer as the polymer neither evaporates nor cures on air alone. Both liquids deform together with the sample, maintaining isolation from air throughout the test. The acetone soaked sample showed maximum temperatures of only $320 \mathrm{~K}$, while the samples covered in uncured polymer showed a slightly higher heating to $360 \mathrm{~K}$ (Figure S7). Fracture occurred by soft pulling apart without any sign of 
increased temperature after fracture and, as expected, no flashes were observed. Apart from isolation from oxygen, both substances also have additional effects such as (1) cooling, either by evaporation of acetone or convective cooling by the liquid polymer and (2) significant reduction of the shear stress between the bundles, as both acetone and the polymer infiltrate the CNT bundle network by wicking.

In summary, for the case of uncoated samples in air, we observe two concomitant effects arising from the combination of a nanoscale, entangled textile morphology and high strain rates: 1) overall frictional heating by bundle sliding, with amplified localized heating at the fracture up to temperatures above CNT decomposition in air, and 2) localized strain rate magnification on the CNT level that leads to a strain energy high enough to cause bond breakage, causing photon emission by recombination.

The flashes are caused by the sample's hierarchical structure and fracture mechanism, and have no detrimental effect on its strength. Further investigation of the phenomenon will include quasi-static tensile tests to prove the strain rate dependence, and a modified setup with a gas enclosure to exclude air, to test for any solid-state response in addition to the observed gas discharge.

Acknowledgements. We thank Kelvin Lim from Dynamic System Analysis Pte Ltd, Singapore, for his support in loaning the 100,000 fps high-speed camera, without which the tests would have required many more samples for hit-and-miss experiments. We thank John S. Bulmer and Adarsh Kaniyoor for their help in CNT sample production and Patrick Kiley, Jeronimo Terrones, and John Bulmer for helpful discussions and their support. This project did not receive any specific grant from funding agencies in the public, commercial, or not-forprofit sectors, however, T. Gspann thanks the Office of Naval Research for visiting scientist support funding (VSP 17-4-002).

Competing interests statement. The authors declare that they have no competing financial interests.

Supplementary Online Material. including high speed camera and IR camera videos accompanies the paper. 


\section{References}

[1] Y.-L. Li, I. A. Kinloch and A. H. Windle, Science, 304(5668), 276-278, 2004.

[2] T. S. Gspann, S. M. Juckes, J. F. Niven, M. B. Johnson, J. A. Elliott, M. A. White and A. H. Windle, Carbon, 114, 160-168, 2017.

[3] A. Mikhalchan, T. Gspann and A. Windle, Journal of Materials Science, 51, 10005-10025, 2016.

[4] N. R. Pradhan, H. Duan, J. Liang and G. S. Iannacchione, Nanotechnology, 20, 245705 (7pp), 2009.

[5] S. Hepplestone, A. Ciavarella, C. Janke and G. Srivastava, Surface Science, 600(18), 3633-3636, 2006.

[6] J. T. Nolan, G. Heymach, Shelley, R. W. Speir, D. H. Buchanan and R. Robbins, The New England Journal of Medicine, 279(6), 323-324, 1968.

[7] L. M. Sweeting, A. L. Rheingold, J. M. Gingerich, A. W. Rutter, R. A. Spence, C. D. Cox and T. J. Kim, Chem. Mater., 9, 1103-1115, 1997.

[8] B. Chandra, Nucl. Tracks, 10, 225-241, 1985.

[9] S. Lebedkin, K. Arnold, F. Hennrich, R. Krupke, B. Renker and M. M. Kappes, New J. Phys., 5, 140 , 2003.

[10] A. Kramida, Y. Ralchenko, J. Reader and a. N. A. Team, "NIST Atomic Spectra Database (ver. 5.3), [Online]," National Institute of Standards and Technology, Gaithersburg, MD., 2015. [Online]. Available: http://physics.nist.gov/asd. [Accessed 10 September 2017].

[11] A. J. Walton, Advances in Physics, 26(6), 887-948, 1977.

[12] M. Barberio, Barone, A. Bonanno, M. Camarca and F. Xu, Radiation Physics and Chemistry, 76(3), 492-494, 2007. 